\title{
LEVANTAMENTO FITOSSOCIOLÓGICO DE PLANTAS DANINHAS EM CULTIVO DE BANANA IRRIGADA
}

\author{
E. R. Moura Filho ${ }^{{ }^{*}}$, L. P. M. Macedo ${ }^{2}$ e A. R. S. Silva ${ }^{1}$
${ }^{1}$ Instituto Federal de Educação, Ciência e Tecnologia do Rio Grande do Norte
${ }^{2}$ Instituto Federal de Educação, Ciência e Tecnologia da Paraíba
edmondson.filho@ifrn.edu.br ${ }^{*}$ \\ Artigo submetido em setembro/2012 e aceito em abril/2015 \\ DOI: 10.15628/holos.2015.1006
}

\section{RESUMO}

Este trabalho objetivou realizar cadastramento fitossociológico das espécies de plantas daninhas na cultura da banana no Vale do Assu, no município de Ipanguaçu-RN. O trabalho foi desenvolvido em uma área cultivada com banana 'maça' em área experimental do IFRN, campus Ipanguaçu. Para realização do estudo, selecionou-se uma área com plantas em plena produção com quatro anos de idade, proveniente do plantio via rizomas. Na caracterização da comunidade infestante foi utilizado o método do quadrado inventário (quadro de $0,5 \times 0,5 \mathrm{~m}$ ), lançado aleatoriamente na área em estudo. Em cada quadro amostrado, as plantas foram identificadas, sendo quantificadas as famílias, os gêneros e as espécies. Para a área estudada, pôde-se observar maior predominância de plantas dicotiledôneas. Foram identificadas 12 espécies distribuídas em 12 gêneros e 9 famílias. A maior infestação foi de espécies da família Poaceae (25\%).

PALAVRAS-CHAVE: Fitossociologia, Manejo de plantas daninhas, bananicultura.

\section{PHYTOSOCIOLOGICAL SURVEY OF WEENDS IN BANANA}

\begin{abstract}
This study aimed to carry out phytosociological registration of weed species in banana cultivation in the Valley of Assu, in the municipality of Ipanguaçu-RN. The study was conducted in an area cultivated with banana 'apple' in the experimental area of IFRN, campus Ipanguaçu. For the study, we selected an area with plants in full production with four years of age, from the planting via rhizomes. In characterizing the weed
\end{abstract}

community was used inventory square method (Table $0.5 \times 0.5 \mathrm{~m})$, released randomly in the study area. In each frame sampled plants were identified and quantified families, genera and species. For the study area, it was observed a predominance of broadleaved plants. 12 species in 12 genera and 9 families were identified. The infestation was most species of the family Poaceae (25\%).

KEYWORDS: Phytosociology, wend control, banana. 


\section{INTRODUÇÃO}

Um dos pontos críticos no processo produtivo da banana é a interferência das plantas daninhas. Essas competem com a cultura por água, luz, nutrientes e gás carbônico, e ainda podem liberar substâncias alelopáticas que podem influenciar no desenvolvimento da cultura, além de hospedar pragas e doenças. Portanto, um manejo adequado das plantas daninhas é de fundamental importância para se obter produtividade na cultura da banana.

Existem diversas plantas daninhas que se desenvolvem associadas à bananicultura, como trapoeraba (Commelina benghalensi), tiririca (Cyperus sp.), bredo (Talinum paniculatum) (Alves, 2005). Essas espécies causam reduções significativas na produção da cultura, exigindo identificação e manejo adequado na convivência com a cultura da banana.

Na região do Vale do Açu, a grande diversidade, a frequência e a abundância de plantas daninhas pouco estudadas são fatores que dificultam o cultivo da banana. Na aplicação de um manejo adequado de plantas daninhas em uma lavoura, exige-se a identificação das espécies e classificação daquelas que têm maior importância. Portanto, o estudo fitossociológico das plantas daninhas, envolvendo parâmetros populacionais como freqüência e abundância são imprescindíveis para determinação e aplicação do manejo das espécies daninhas. A cobertura vegetal sofre influência do clima, solo e fauna, cuja comunidade é caracterizada por grupos de populações que variam em espécie, fluxo de emergência, índice de mortalidade, taxa de crescimento absoluto e ciclo de desenvolvimento (Gomes et. al., 2010).

Sendo assim, o objetivo deste trabalho foi realizar o levantamento fitossociológico das espécies de plantas daninhas na cultura da banana no Vale do Açu.

\section{MATERIAL E MÉTODOS}

O trabalho foi desenvolvido durante o mês de julho de 2010, em uma área cultivada com banana 'maçã' dentro do campus do IFRN de Ipanguaçu, município de Ipanguaçu-RN, cujas coordenadas geográficas são de $5^{\circ} 29^{\prime} 56^{\prime \prime}$ de latitude sul e uma longitude oeste de $36^{\circ} 51^{\prime} 10^{\prime \prime}$ e altitude média de $13 \mathrm{~m}$. O clima da região, de acordo com a classificação de Koeppen, é do tipo BSwh', ou seja, quente e seco, com precipitação pluviométrica bastante irregular, com média anual de $903,3 \mathrm{~mm}$, temperatura média anual de $27,9^{\circ} \mathrm{C}$ e umidade relativa do ar média de $70 \%$ (Costa e Silva, 2008).

O solo da área experimental foi classificado como um Neossolo Flúvico (Santos, et al., 2006), textura franco arenosa, com $9 \%$ de argila, $26 \%$ de silte, $9 \%$ de areia grossa e $56 \%$ de areia fina, cujas principais características químicas são: $\mathrm{pH}$ em água $=8,1 ; \mathrm{P}=76,2 \mathrm{mg} \mathrm{dm}^{-3} ; \mathrm{K}=46,63$ $\mathrm{mg} \mathrm{dm}{ }^{-3} ; \mathrm{Ca}=3,3 \mathrm{cmolc} \mathrm{dm}^{-3} ; \mathrm{Mg}=1,70 \mathrm{cmolc} \mathrm{dm}^{-3} ; \mathrm{H}+\mathrm{Al}=0,50 \mathrm{cmolc} \mathrm{dm}{ }^{-3} ; \mathrm{MO}=10,7 \mathrm{~g} \mathrm{Kg}^{-1}$.

Para realização do estudo, selecionou-se uma área com plantas em plena produção com quatro anos de idade, proveniente do plantio via rizomas. Na caracterização da comunidade infestante foi utilizado o método do quadrado inventário (quadro de 0,5 x 0,5 m), lançado 15 aleatoriamente na área em estudo. Em cada quadro amostrado, as plantas foram identificadas, sendo quantificadas as famílias, os gêneros e as espécies; em seguida, foi cortada a parte aérea, que foram acondicionadas em sacos de papel para encaminhamento ao laboratório, onde foram 
secas em estufa de circulação forçada de ar e mantidas a $60^{\circ} \mathrm{C}$ por 72 horas, para, em seguida ser realizada a pesagem do material, em balança de precisão.

A identificação e contagem das espécies permitiu calcular as seguintes variáveis fitossociológicas: frequência, densidade, frequência relativa, densidade relativa, índice de valor de importância e índice de importância relativa.

Nos cálculos dessas variáveis foram utilizadas as seguintes fórmulas:

- Frequência $(F)=n^{\circ}$ de quadrados que contêm a espécie $\div n^{\circ}$ total de quadrados obtidos (área total). Os resultados obtidos permitem avaliar a distribuição das espécies nas parcelas.

- Densidade $(D)=n^{\circ}$ total de indivíduos por espécie $\div n^{\circ}$ total de quadrados obtidos (área total). Permite gerar dados sobre a quantidade de plantas de cada espécie por unidade de área.

- Frequência Relativa $(F r)=100 \times$ frequência da espécie $\div$ frequência total de todas as espécies.

- Densidade Relativa $(\mathrm{Dr})=100 \times$ densidade da espécie $\div$ densidade total de todas as espécies;

- As variáveis Fr e Dr permitem obter informações sobre a relação de cada espécie com as outras espécies encontradas na área.

- Índice de Valor de Importância (IVI) = frequência relativa + densidade relativa + abundância relativa.

- Importância Relativa (IR) = $100 \mathrm{x}$ índice de valor de importância da espécie $\div$ índice de valor de importância total de todas as espécies.

\section{RESULTADOS E DISCUSSÃO}

Para a área estudada, pôde-se observar maior predominância de plantas dicotiledôneas. Foram identificadas 12 espécies distribuídas em 12 gêneros e 9 famílias (Tabela 1). A família que apresentou maior número de espécies na área de estudo foi a Poaceae, com 3 espécies, perfazendo um total de 25\%, Corroborando os resultados obtidos por Gomes et al. (2010), que em área de várzea cultivada com banana a família que apresentou maior número de espécies foi a Poaceae, perfazendo um total de $33 \%$.

Tabela 1 - Relação de plantas daninhas, distribuídas por família e espécie, ocorrentes na área de banana, no município de Ipanguaçu - RN.

\begin{tabular}{|l|l|l|l|}
\hline \multicolumn{1}{|c|}{ Família } & \multicolumn{1}{c|}{ Gênero } & \multicolumn{1}{c|}{ Nome científico } & \multicolumn{1}{c|}{ Nome comum } \\
\hline Leguminosae & Senna & Senna obtusifolia L. & Fedegoso \\
\hline Poaceae & Dactyloctenium & Dactyloctenium aegyptium L. & Capim mão de sapo \\
\hline Amaranthaceae & Alternanthera & Alternanthera tenella Colla & Apaga fogo \\
\hline Asteraceae & Emilia & Emilia sonchifolia L. & Serralhinha \\
\hline Rubiaceae & Richardia & Richardia brasiliensis Gomes & Poaia \\
\hline Commelinaceae & Commelina & Commelina benghalensis L. & Trapoeraba \\
\hline Poaceae & Eragrotis & Eragrotis pilosa & Eragrostis \\
\hline Convolvulaceae & Merremia & Merremia aegyptia & Jitirana \\
\hline
\end{tabular}




\begin{tabular}{|l|l|l|l|}
\hline Turneraceae & Turnera & Turnera ulmifolia & Chanana \\
\hline Cyperaceae & Cyperus & Cyperus rotundus L. & Tiririca \\
\hline Leguminosae & Phaseolus & Phaseolus lathiroides & Feijão-de-rola \\
\hline Poaceae & Digitaria & Digitaria sanguinalis L. & capim milhã \\
\hline
\end{tabular}

No cultivo da banana, as principais espécies encontradas foram: Cyperus rotundus, com densidade de 7,13 plantas $\mathrm{m}^{-2}$, e 0,333 de frequência; Commelina benghalensis, com densidade de 3,80 plantas $\mathrm{m}^{-2}$ e 0,333 de frequência; Emilia sonchifolia, com densidade de 3,47 planta $\mathrm{m}^{-2}$, 0,667 de frequência; Alternanthera tenella, com densidade de 1,33 plantas $\mathrm{m}^{-2}$ e 0,600 de frequência; Richardia brasiliensis, com densidade 1,20 plantas $\mathrm{m}^{-2}, 0,467$ de frequência (Tabela 2).

Tabela 2 - Número de quadrados onde a espécie foi encontrada, número de indivíduos, freqüência e densidade de espécies daninhas em cultivo de banana, no município de Ipanguaçu - RN.

\begin{tabular}{|l|c|c|c|c|}
\hline \multicolumn{1}{|c|}{ Espécies } & $\begin{array}{c}\text { Número de } \\
\text { quadrados } \\
\text { com } \\
\text { ocorrência }\end{array}$ & $\begin{array}{c}\text { Número } \\
\text { de } \\
\text { indivíduos }\end{array}$ & Frequência & $\begin{array}{c}\text { Densidade } \\
\text { (plantas } \mathrm{m}^{-2} \text { ) }\end{array}$ \\
\hline Senna obtusifolia L. & 1 & 1 & 0,067 & 0,07 \\
\hline Dactyloctenium aegyptium L. & 1 & 5 & 0,067 & 0,33 \\
\hline Alternanthera tenella Colla & 9 & 20 & 0,600 & 1,33 \\
\hline Emilia sonchifolia L. & 10 & 52 & 0,667 & 3,47 \\
\hline Richardia brasiliensis Gomes & 7 & 18 & 0,467 & 1,20 \\
\hline Commelina benghalensis L. & 5 & 57 & 0,333 & 3,80 \\
\hline Eragrotis pilosa & 5 & 6 & 0,333 & 0,40 \\
\hline Merremia aegyptia & 1 & 2 & 0,067 & 0,13 \\
\hline Turnera ulmifolia & 5 & 3 & 0,333 & 0,20 \\
\hline Cyperus rotundus L. & 5 & 107 & 0,333 & 7,13 \\
\hline Phaseolus lathiroides & 5 & 6 & 0,333 & 0,40 \\
\hline Digitaria sanguinalis L. & 1 & 1 & 0,067 & 0,07 \\
\hline
\end{tabular}

$\mathrm{Na}$ área em estudo, destacam-se, em termos de Densidade Relativa (\%) e Frequência Relativa (\%), as espécies Cyperus rotundus, Commelina benghalensis e Emilia sonchifolia (Figura 1). A espécie $C$. rotundus é uma espécie bastante prejudicial à bananicultura, pois se reproduz com facilidade, dominando as áreas rapidamente. Isso tem aumentado a importância dessa espécie na comunidade infestante, onerando a atividade de produção da banana, devido ao aumento dos custos para seu controle (Gomes et al., 2010).

Vários autores (Maxwell; Ghersa, 1992; Wiles et al., 1992) constataram que as reduções de produtividade impostas pelas plantas daninhas dependem da densidade média e, também, da distribuição delas no campo. Esses autores salientaram que o desenvolvimento de sistemas de manejo integrado que considerem também a distribuição das plantas daninhas tem sido limitado pela ausência de maiores estudos. 


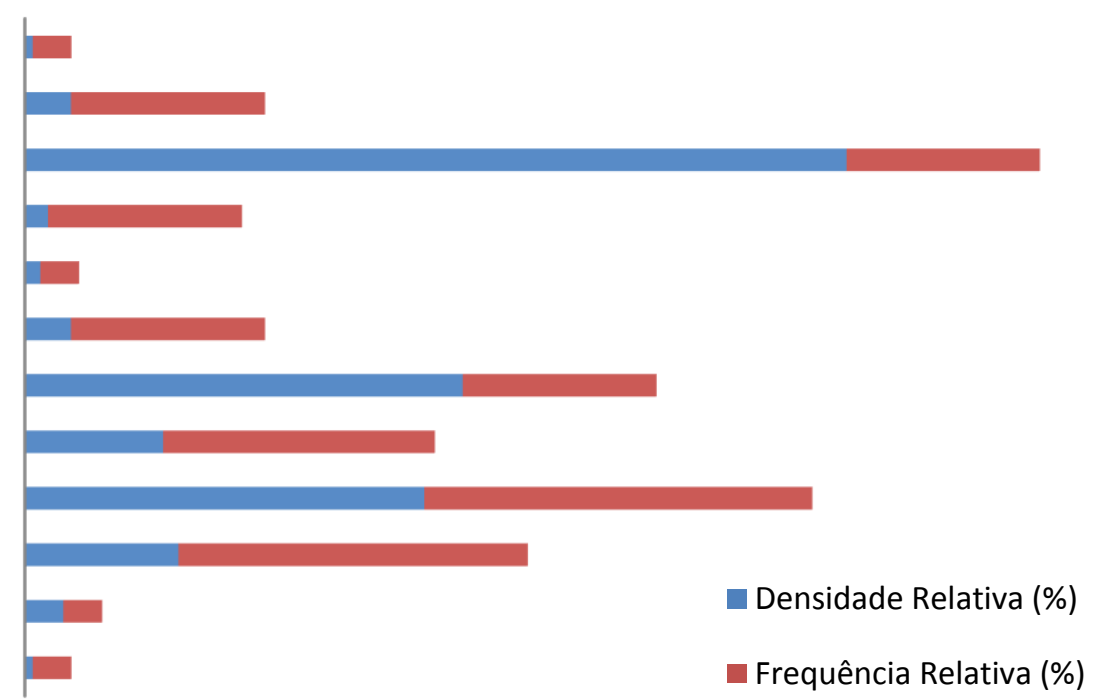

Figura 1 - Densidade relativa e frequência relativa das principais espécies de plantas daninhas na cultura da banana, no município de Ipanguaçu - RN.

Na Tabela 3 são apresentados os dados referentes ao Índice de Valor de Importância (IVI) e Importância Relativa (IR) das plantas daninhas infestantes na área com bananicultura.

$\mathrm{Na}$ área em estudo, as populações que apresentaram os maiores IVI, foram: Alternanthera tenella, Emilia sonchifolia, Cyperus rotundus. Já a IR, as espécies de maior importância relativa foram: Alternanthera tenella, Emilia sonchifolia, Cyperus rotundus, Commelina benghalensis. Já a espécie com os menores valores de IVI e IR foi Digitaria sanguinalis (Tabela 5). A importância relativa infere quais espécies são mais importantes em termos de infestação (Pitelli, 2000), levando em consideração a distribuição das espécies, o número de indivíduos e a concentração na área.

Tabela 3 - Índice de valor de importância e importância relativa das plantas daninhas na cultura da banana, no município de Ipanguaçu - RN.

\begin{tabular}{|l|c|c|}
\hline \multicolumn{1}{|c|}{ Espécies } & $\begin{array}{c}\text { Índice de Valor de } \\
\text { Importância (IVI) }\end{array}$ & Importância Relativa (IR) \\
\hline Senna obtusifolia & 4,512 & 1,504 \\
\hline $\begin{array}{l}\text { Dactyloctenium } \\
\text { aegyptium }\end{array}$ & 5,859 & 1,953 \\
\hline Alternanthera tenella & 70,032 & 23,344 \\
\hline Emilia sonchifolia & 64,787 & 21,596 \\
\hline Richardia brasiliensis & 20,090 & 6,697 \\
\hline Commelina benghalensis & 43,228 & 14,409 \\
\hline Eragrotis pilosa & 11,570 & 3,857 \\
\hline Merremia aegyptia & 2,577 & 0,859 \\
\hline Turnera ulmifolia & 13,082 & 4,361 \\
\hline Cyperus rotundus & 50,253 & 16,751 \\
\hline Phaseolus lathiroides & 11,817 & 3,939 \\
\hline Digitaria sanguinalis & 2,194 & 0,731 \\
\hline
\end{tabular}


As populações de maior importância relativa não foram prejudicadas pelo manejo de controle de plantas daninhas, pois, segundo Pitelli (2000), as espécies indiferentes ou favorecidas por práticas agrícolas tendem a apresentar percentuais mais elevados. Essas plantas infestantes ocorreram por todo o período avaliado, apresentando-se com diferentes estádios fenológicos, desde a germinação até a floração e formação de sementes.

\section{CONCLUSÕES}

De forma geral, a área em estudo apresentou baixa infestação de plantas daninhas e comunidades muito distintas. Sendo as mais frequentes e com maior densidade as espécies Cyperus rotundus, Commelina benghalensis e Emilia sonchifolia. A espécie Alternanthera tenella apresentou os maiores índices de valor de importância e de importância relativa na área.

\section{REFERÊNCIAS BIBLIOGRÁFICAS}

1. ALVES, E. Plantas daninhas na cultura da banana (Musa sp.). In: REUNIÃO ITINERANTE DE FITOSSANIDADE DO INSTITUTO BIOLÓGICO: CULTURA DA BANANA, 13., 2005, Registro. Anais... Registro: Apta Regional Vale do Ribeira, 2005. p. 54-60.

2. COSTA, J.R.S.; SILVA, F.M; Análise da precipitação na cidade de Ipanguaçu/RN por imagens de satélite e distribuição de gumbel. In: SIMPÓSIO BRASILEIRO DE GEOGRAFIA FÍSICA APLICADA, XIII, 2008, Viçosa-MG, Anais..., Viçosa-MG: 2008.

3. GOMES, G.L.G.C.; IBRAHIM, F.N.; MACEDO, G.L.; NOBREGA, L.P.; ALVES, E. Cadastramento fitossociológico de plantas daninhas bananicultura. Planta Daninha, Viçosa-MG, v. 28, n. 1, p. 61-68, 2010.

4. MAXWELL, B. D.; GHERSA, C. The influence of weed seed dispersion versus the effect of competition on crop yield. Weed Technol., v. 6, n. 1, p. 196-204, 1992.

5. PITELLI, R. A. Estudos fitossociológicos em comunidades infestantes de agroecossistemas. J. Conserb, v. 1, n. 2, p. 17, 2000.

6. WILES, L. J. et al. Spatial distribuition of broadleaf weeds in North Carolina soybean (Glycine max) field. Weed Sci., v. 40, p. 554-557, 1992. 